\title{
Real-Time Optimal Scheduling of Smart Power Distribution Systems Using Integrated Receding Horizon Control and Convex Conic Programming
}

\author{
Seyedmahdi Moghadasi, Student Member IEEE, Sukumar Kamalasadan, Member IEEE \\ Power, Energy and Intelligent Systems Laboratory \\ Department of Electrical and Computer Engineering \\ University of North Carolina at Charlotte, Charlotte, NC \\ smoghada@uncc.edu,skamalas@uncc.edu
}

\begin{abstract}
In this paper, we propose a convex Optimal Power Flow (OPF) formulation integrated within Receding Horizon Control (RHC) method using Second Order Conic Programming (SOCP). The main advantages of the proposed method are a) global optimal scheduling with faster computation time b) dynamic equation models with real-time control c) integration of uncertain resources and measurements. The effectiveness of this method is evaluated using a 32 bus power distribution test system, considering various network constraints that include market interaction, energy storage dynamics and uncertain model of wind generation. The efficiency of the proposed method compared to RHC AC Optimal Power Flow (RHC-ACOPF) architecture is also evaluated. The results show that the proposed architecture outperforms the RHC-ACOPF in computation time and guaranties the global optimal solution. Also the proposed method provides effective usage of energy storage system as the RHC integration allows including dynamic modeling of energy storage within the optimization algorithm.
\end{abstract}

Index Terms-Convex Optimization, Second Order Conic Programming (SOCP), Semidefinite Programming (SDP), Receding Horizon ControL (RHC), Real-time Optimization, Electricity Market, Radial Distribution System.

\section{NOMENCLATURE}

Indices

$i, j, h$

nodes of radial systems

$k, T$ time step and horizon time

Variables and Parameters

$T(N, E)$

$S_{i j}, P_{i j}, Q_{i j}$

$R_{i j}, X_{i j}$

$B_{i}, G_{i}$

$I_{i j}$

$\lambda_{i j}$

$v_{i j}$

$\omega_{i j}$

$B_{\perp}$

$\beta_{\perp}$

$\theta_{*}$

$P_{w g, i}^{k}, P_{d g, i}^{k}$

$P_{c l, i}^{k}$

$P_{m, i}^{k}, P_{n d, i}^{k}$

$c_{W G, i}^{k}, c_{D G, i}^{k}$

$c_{C L, i}^{k}$
$c_{M, i}^{k}, c_{N D, i}^{k}$

$E_{s, i}^{k}$

$P_{c h, i}^{k, i}, P_{d i c h, i}^{k}$

$\eta_{\text {ch,i }}, \eta_{\text {dich }, i}$

ShortUsage

ACOPF

SOCP-OPF

RHC-ACOPF

RHC-SOCP-OPF wholesale and neighboring price energy storage at time step $k$ charging and discharging rates charging and discharging efficiency

Non-convex OPF

Convex Conic OPF

ACOPF integrated in RHC

Convex conic OPF integrated in RHC

\section{INTRODUCTION}

$\mathbf{F}$ AST optimal scheduling of power grid is now an important requirement for economically integrating and operating electric power network which increasingly incorporate inherent stochastic players such as renewable sources, plugin electric vehicles (PEV), price responding demands, and electricity market interactions [1]. Optimal power flow (OPF), evolved in 1962 [2], formulated the basic problem of finding a local optimum operating point for a power system given that both the demand and supply are deterministic. From then on, numerous studies have proposed optimization algorithms to solve OPF problem more efficiently. All these algorithms carry with them various well known limitations referred to as the non-exact relaxation and locally optimum solution [3]- [6]. These limitations are mainly resulting from nonconvexity of OPF equations [7]. In recent years, significant contributions are observed in convexification of OPF in radial networks. In 2006, ref [8] proposed the convexification of branch flow OPF for radial system using second order conic programming (SOCP) relaxation. The branch flow OPF [9][10] for optimal capacitor placement in distribution systems, exploited the voltage and current angle relaxation; however, this model was non-convex as a result of quadratic equality constraints. Several continuous convex relaxation of this model were then propounded using second order cone approximation [11]- [12]. In important studies, [13]- [15] observed and proved the exactness of angle and conic relaxation in convexification, providing an exact optimal solution for OPF problem in radial networks. A distributed optimal power flow using semi- 
definite programming [16] also evaluates the exactness of SDP relaxation of bus injection OPF model in distribution system. A significant proof for zero duality gap presented in [7] shows that strong duality condition exists for the proposed dual OPF using bus injection model and SDP.

Distribution systems gradually transform from a passive network to an active and smart network as a result of evolving rapid distributed energy resources (DER), storage facilities alongside with renewable supplies and electricity markets, which increase the degree of freedom on OPF problem and necessitate dealing with uncertainties through a real-time optimization [17]. Receding horizon control (RHC) [18] is a promising method to schedule the control variables over a time horizon divided by small time steps which allows for realtime implementation. In our earlier work, we proposed and examined the framework of a real-time ACOPF integrated in RHC [19] to re-forecast the future market price and incorporate this updates in our dynamic OPF, considering power and physical network constraints. Ref. [20] proposed the iterative method of prox-average message passing to decentralize the optimization over devices and suggest RHC as an extension to the approach. Recently [21] examined RHC-ACOPF to find better DGs set-point in distribution system. A parallel multistage dispatch using RHC propounded by [22] evaluated the control feasibility of network layers for bulk power system. Other studies have investigated the RHC in scheduling of deferable electric loads without power constraints [23], and scenario-based RHC for optimal power dispatch [24]. Nevertheless, there are two major drawbacks of using RHC with nonlinear ACOPF: computational burden at each time step and also the locally optimum solution.

The studies presented so far focus on either the OPF convexification or RHC method independently. In this paper, we extend our proposed real-time framework RHC-ACOPF [19] by incorporating a convex conic OPF within the RHC (RHC-SOCP-OPF). This method results in finding the optimal schedule, and at the same time brings significant saving in computation time due to efficient convex optimization algorithms compared with a non-convex optimization in ACOPF. The proposed method could also include dynamic device models in the minimization problem such as energy storage and at the same time allows for including measurements and other stochastic variables. To the best of our knowledge, it is the first time that a method introduces the convex OPF integrated in RHC. The proposed method is tested on a modified IEEE distribution system, equipped with various constraints and components associated with a smart micro grid.

Rest of the paper is organized in the following order. III-A presents the RHC formulation dealing with OPF problem, and III-B discusses the convexification of OPF problem using SOCP. Section IV formulate the real-time convex OPF. Implementation of the developed method on the 32-bus distribution test system and the study results are presented in section V. The case study contains market interactions including neighboring distribution companies (DISCOs) and upstream network, uncertain wind generation, storage, distributed energy resources, and contracted loads. Study results evaluate the effectiveness of convex RHC-SOCP-OPF compared with
RHC-ACOPF. Finally, conclusion is presented in section VI.

\section{System ARChitecture AND Modeling}

The proposed real-time convex OPF architecture consists of two major sections, RHC as the master control and convex conic OPF as the internal slave.

\section{A. RHC Methodology}

RHC output is a set of consecutive control actions, created by minimizing the objective function over a horizon window. The process has the capability of dealing with constrained dynamic optimization problems which includes consideration of uncertain inputs or parameters. In dynamic systems, state update is captured by (1) whereby the dynamic matrix $\hat{A}_{(k \mid t)}$ and input matrix $\hat{B}_{(k \mid t)}$ is determined based on the real-time information available at the current step. The control actions $\hat{u}_{(k \mid t)}$ are the solution of the optimization problem (2) [18].

$$
\begin{aligned}
& \hat{A}_{(k+1 \mid t)}=\hat{A}_{(k \mid t)} \hat{x}_{(k \mid t)}+\hat{B}_{(k \mid t)} \hat{u}_{(k \mid t)} \\
& \operatorname{Min} \sum_{k=t}^{t+T} \hat{J}_{(k \mid t)}\left(\hat{x}_{(k \mid t)}, \hat{u}_{(k \mid t)}\right) \\
& \text { Sub. } \hat{A}_{(k+1 \mid t)}=\hat{A}_{(k \mid t)} \hat{x}_{(k \mid t)}+\hat{B}_{(k \mid t)} \hat{u}_{(k \mid t)} \\
& \left(\hat{x}_{(k \mid t)}, \hat{u}_{(k \mid t)}\right) \in \hat{C}_{(k \mid t)} \quad k=t, \ldots, T
\end{aligned}
$$

The control procedure comprises of the following steps:

1) The constrained objective function $\hat{J}_{(k \mid t)}$ is defined over the time horizon $[t: t+T-1]$. The system parameters and constraints $\hat{C}_{(k \mid t)}$ are included based on the real-time data available until time step $t$.

2) The optimization problem (2) is solved, and the system control variables is determined over the horizon window $[t: t+T-1]$. Then the system states $\hat{x}_{(k \mid t)}$ and control actions $\hat{u}_{(k \mid t)}$ is passed on to the system.

3) Feedback of system variables and external signals such as market price and new weather forecast are passed on back to the controller to update the system states and these inputs are re-forecasted over the next horizon window $[t+1: t+T]$.

4) The system moves into the next time step $t+1$, and $[t+1: t+T]$ becomes the new horizon window. The preceding steps are repeated over the new window.

The RHC method continues until the time step reach the horizon $T$. Handing the time horizon is a significant feature in the proposed approach which brings in the ability to include uncertain parameters and real-time system feedback. Thus this method creates the platform to handle the optimization incorporating dynamic components such as storage in power distribution system. Uncertainty is generally modeled using stochastic models based on random variables. We developed (3)-(5) to forecast the random variable $\hat{z}$ over the horizon window $[k: k+T-1]$ using real-time data $z_{t}, z_{t-1}, \cdots, z_{t-N+1}$ with the conditional expectation expression. The linear regression method is deployed to generate the forecast values. i.e. the conditional expectation of random variable at time step $k\left(\hat{z}_{k \mid t}\right)$ is estimated as linear function of the last $N$ real-time feedback data, $Z=\left[z_{t}, z_{t-1}, \cdots, z_{t-N+1}\right]$, density 


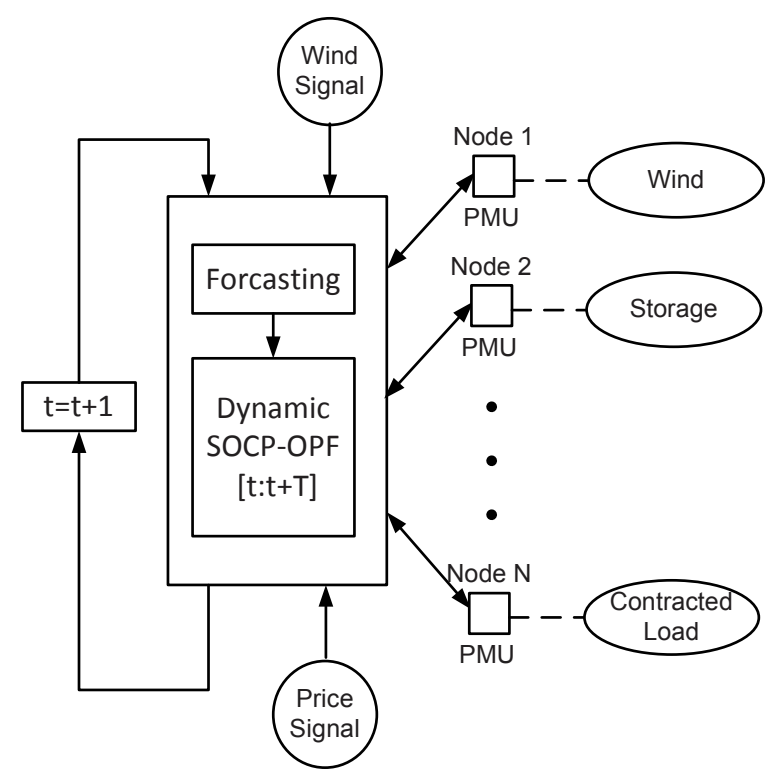

Fig. 1. RHC Dealing With OPF in Modern Power Grid

function expectation $E\{z\}$ and variance $E\left\{z_{t}-E\left\{z_{t}\right\}\right\}^{2}$. This recursive formulation deals with random variables in which the variables have a normal joint distribution.

$$
\begin{gathered}
\hat{z}_{(k \mid t)}=E\left(z_{k} \mid z_{t}, z_{t-1}, \cdots, z_{t-N+1}\right)=\alpha+\beta^{T} Z \\
\alpha=E\left\{z_{k}\right\}-\beta^{T}\left[\begin{array}{c}
E\left\{z_{t}\right\} \\
E\left\{z_{t-1}\right\} \\
E\left\{z_{t-2}\right\} \\
\vdots \\
E\left\{z_{t-N+1}\right\}
\end{array}\right] \\
\beta=\left[\begin{array}{c}
\frac{E\left\{\left(z_{t}-E\left\{z_{t}\right\}\right)\left(z_{k}-E\left\{z_{k}\right\}\right)\right\}}{E\left\{z_{t}-E\left\{z_{t}\right\}\right\}^{2}} \\
\frac{E\left\{\left(z_{t-1}-E\left\{z_{t-1}\right\}\right)\left(z_{k}-E\left\{z_{k}\right\}\right)\right\}}{E\left\{z_{t-1}-E\left\{z_{t-1}\right\}\right\}^{2}} \\
\frac{E\left\{\left(z_{t-2}-E\left\{z_{t-2}\right\}\right)\left(z_{k}-E\left\{z_{k}\right\}\right)\right\}}{E\left\{z_{t-2}-E\left\{z_{t-2}\right\}\right\}^{2}} \\
\vdots \\
\frac{E\left\{\left(z_{t-N+1}-E\left\{z_{t-N+1}\right\}\right)\left(z_{k}-E\left\{z_{k}\right\}\right)\right\}}{E\left\{z_{t-N+1}-E\left\{z_{t-N+1}\right\}\right\}^{2}}
\end{array}\right]
\end{gathered}
$$

The forecasted values $Z=\left[z_{t}, z_{t-1}, \cdots, z_{t-N+1}\right]$ resulting from (3)-(5) is then used in RHC optimization (2) to obtain the optimal control variables $\hat{x}_{(k \mid t)}, \hat{u}_{(k \mid t)}, k \in[t, t+T-1]$ which minimizes the cost function over the next time horizon $T$. The process is repeated at the next time step for updates. Fig. 1 depicts the forecasting section inside the RHC structure dealing with SOCP-OPF problem. Convexification method is discussed next.

\section{B. Convex conic $O P F$}

The convex OPF is a critical requirement to reach an optimal solution with a lower computation time. The proposed
SOCP-OPF is formulated based on branch flow (BF) model. The branch flow model is the method of modeling power flow problem using branch variables such as line current and line power unlike the nodal variables in the bus injection method. The model is described based on a directed tree graph denoted as $T(N, E)$ representing the radial distribution system that starts with Node 0 which is connected to the upstream network. The BF model equations are demonstrated in equation set (6). Notations are given in the nomenclature.

$$
\begin{aligned}
& S_{i j}=V_{i} I_{i j}^{*} \\
& V_{i}-V_{j}=z_{i j} I_{i j} \\
& S_{j}=\sum_{h} S_{j h}-\sum_{i}\left(S_{i j}-z_{i j}\left|I_{i j}\right|^{2}\right)+y_{j}^{*}\left|V_{j}\right|^{2} \\
& \forall\{i, j, h,(i, j),(j, h)\} \in T(N, E)
\end{aligned}
$$

The OPF model based on BF, is a non-convex optimization problem due to nonlinear equality constraints. The relaxation method consisting of two consecutive stages is exploited to convert the problem to a convex optimization problem [15]. In the first stage, the angle relaxation step consist of eliminating angle of all bus voltages and line currents yielding the angle relaxed OPF [9]. In the second stage referred as the conic relaxation, the conic equality constraints are relaxed to conic inequality constraints. These relaxations converts the ACOPF problem to the SOCP-OPF problem, making it as a convex optimization problem. This convexified SOCP-OPF is demonstrated in (7).

$$
\begin{aligned}
& \operatorname{Min} \sum_{i \in N_{G}} c_{i}\left(P_{g}^{i}\right) \\
& P_{j}=\sum_{h} P_{j h}-\sum_{i}\left(S_{i j}-R_{i j} \lambda_{i j}\right)+G_{j} \omega_{j} \\
& Q_{j}=\sum_{h} Q_{j h}-\sum_{i}\left(S_{i j}-X_{i j} \lambda_{i j}\right)+B_{j} \omega j \\
& \omega j=\omega i-2\left(R_{i j} P_{i j}+X_{i j} Q_{i j}\right)+\left(R_{i j}^{2}+X_{i j}^{2}\right) \lambda_{i j} \\
& \lambda_{i j} \leq \frac{P_{i j}^{2}+Q_{i j}^{2}}{\omega i}
\end{aligned}
$$

These relaxations are exact; i.e. the solution of the SOCPOPF (7) gives the optimal solution to the ACOPF problem. (7) yields the magnitude of bus voltage and line current. OPF convexification algorithm is as shown in Fig. 2. The final step is the angle recovery of voltages and currents using the angle recovery algorithm. With this the reduced incidence matrix $B_{m x n}$ and the phase angle difference $\beta_{i j} \forall(i, j)$ are calculated and the voltage angle are then recovered through (9). The necessary condition of angle recovery (8) always holds for radial networks [15];

$$
\begin{aligned}
& B_{\perp} B_{T}^{-1} \beta_{\perp}-\beta_{\perp}=0 \\
& \theta_{*}=\operatorname{Res} .\left(B_{T}^{-1} \beta_{T}\right) \in(-\pi, \pi)
\end{aligned}
$$

The other variables angles are then recoverable using Ohm's law and power injection equation.

\section{Problem Formulation OF RHC-SOCP-OPF}

We developed the RHC-SOCP-OPF formulation to find the global optimum to the OPF problem in radial distribution systems in real-time. The whole structure of proposed 


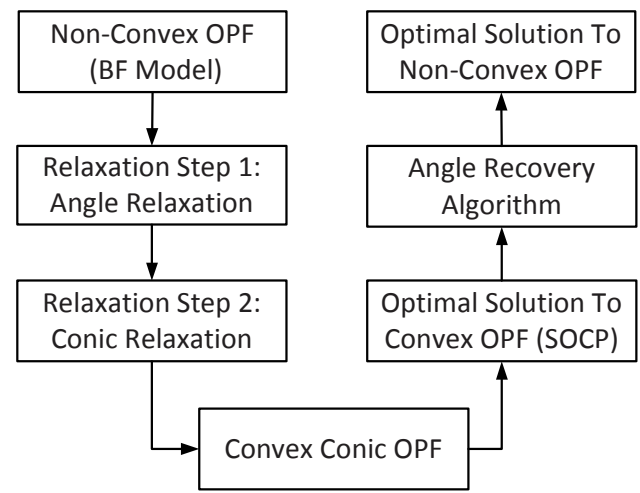

Fig. 2. OPF Convexification: Two Step Relaxation

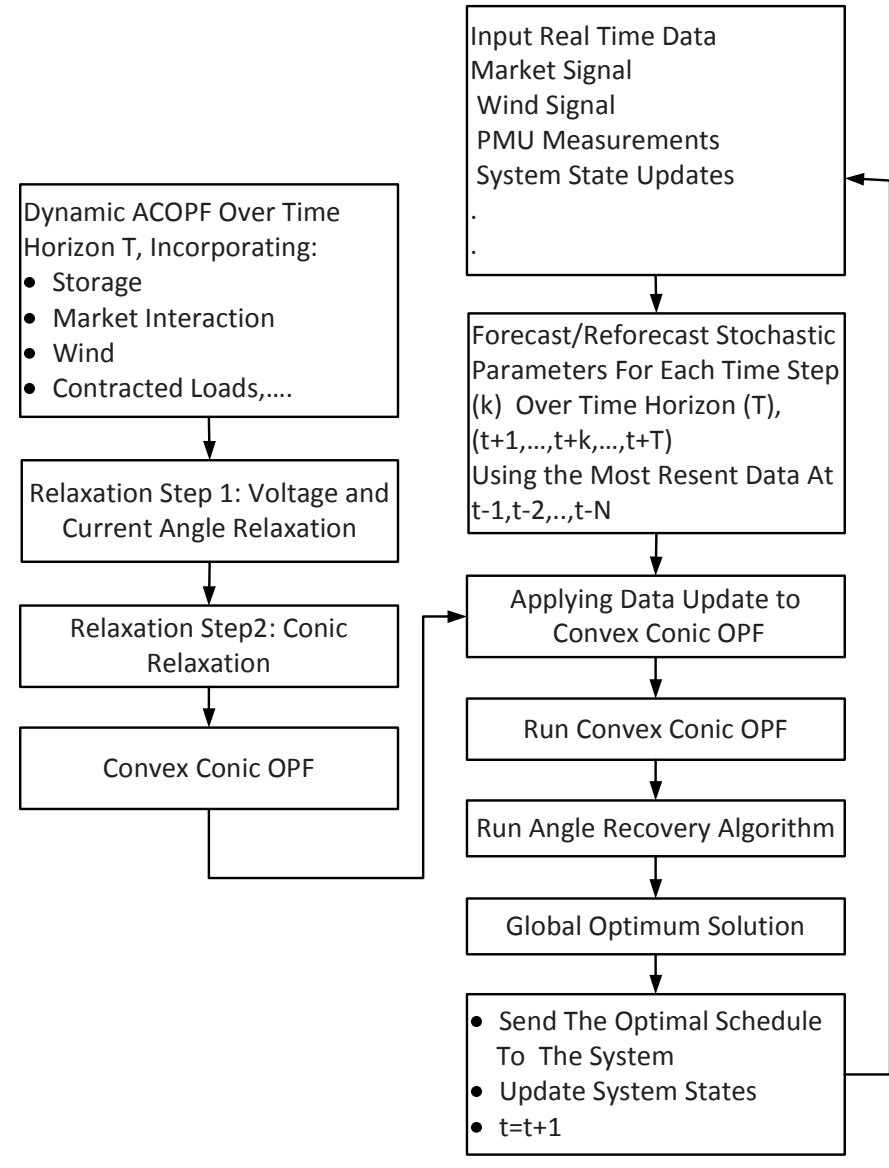

Fig. 3. Optimal Scheduling using Convex RHC

method are depicted in Fig. 3. In order to implement an RHC optimization structure, The first step is to formulate the RHC-ACOPF problem for the system including the storage, wholesale market, neighboring DISCO, and wind generations.

This OPF is based on the BF model and formulated over the time horizon. In order to reach optimal solution and improving computation time, two relaxation steps are implemented: angle relaxation and conic relaxations; The resulting optimization problem is a convex conic optimization problem over the time horizon $T$. The problem formulation is described below.

\section{A. Objective Function}

The objective function takes into account the generation cost, contracted load cost and the cost for energy exchanged with upstream network and neighboring DISCO. The cost function at the time step $t$ are extended in (10). The distribution system here is considered as a micro-grid with the capability of exchanging power with the rest of grid, and the objective function is the total operation cost of the system.

$$
\begin{gathered}
\sum_{k=t}^{t+T-1} \sum_{i \in N}\left(c_{W G, i}^{k}\left(P_{w g, i}^{k}\right)+c_{D G, i}^{k}\left(P_{d g, i}^{k}\right)+c_{C L, i}^{k}\left(P_{c l, i}^{k}\right)\right. \\
\left.+c_{M, i}^{k}\left(P_{m, i}^{k}\right)+c_{N D, i}^{k}\left(P_{n d, i}^{k}\right)\right) \\
k \in[t: t+T-1]
\end{gathered}
$$

\section{B. Constraints}

The power flow equations are the physical laws constraining the path of electricity in the grid, and dictating the real-time balance between active (reactive) power generation and active (reactive)demand. Equation(11) represents the relaxed power balance constraints over the horizon window.

$$
\begin{aligned}
P_{w g, i}^{k}+P_{d g, i}^{k}+P_{c l, i}^{k} & +P_{c h, i}^{k}-P_{d c h, i}^{k}-P_{d, i}^{k} \\
& =\sum_{h} P_{i h}^{k}-\sum_{j}\left(S_{j i}^{k}-R_{j i}^{k} \lambda_{j i}^{k}\right)+G_{i}^{k} \omega i^{k} \\
Q_{w g, i}^{k}+Q_{d g, i}^{k}+Q_{c l, i}^{k} & -Q_{d, i}^{k} \\
& =\sum_{h} Q_{i h}^{k}-\sum_{j}\left(S_{j i}^{k}-X_{j i}^{k} \lambda_{j i}^{k}\right)+B_{i}^{k} \omega i^{k}
\end{aligned}
$$

$k \in[t: t+T-1]$

The voltage drop constraint over the power lines are formulated through Ohm's law and is relaxed using angle relaxation method. This constraint is shown in (12).

$$
\begin{aligned}
& \omega i^{k}=\omega j^{k}-2\left(R_{j i}^{k} P_{j i}^{k}+X_{j i}^{k} Q_{j i}^{k}\right)+\left(R_{j i}^{k^{2}}+X_{j i}^{k}\right) \lambda_{j i}^{k} \\
& k \in[t: t+T-1]
\end{aligned}
$$

The total power flow through the line is defined by the bus voltage and line current. This constraint is relaxed (13) using conic relaxation.

$$
\begin{aligned}
& \lambda_{i j}^{k} \leq \frac{P_{i j}^{k^{2}}+Q_{i j}^{k^{2}}}{\omega i^{k}} \\
& k \in[t: t+T-1]
\end{aligned}
$$

Storage components impose dynamic constraints to the optimization problem. The state of energy stored in the storage at each time step is dictated by the preceding energy state, charging rate, and discharging rate. These are also known as efficiency coefficients representing the efficiency of charging and discharging process. Equation (14) represents this dynamic equation.

$$
\begin{aligned}
& E_{s, i}^{k+1}=E_{s, i}^{k}+P_{c h, i}^{k} \eta_{c h, i}-P_{d i c h, i}^{k} / \eta_{d i c h, i} \\
& k \in[t: t+T-1]
\end{aligned}
$$

The RHC structure creates the capability to update the constraints associated with distribution lines, generation, demand and storage at each time step as depicted in fig 3, despite the

\section{U.S. Government work not protected by U.S. copyright}


conventional day-ahead scheduling which contains a constant limit on each time step. Equation (15) represents these constraints at time step $k$. The expected wind generation and spot market price is also updated at each time step over the next horizon window.

$$
\begin{aligned}
& P_{w g . \min , i}^{k} \leq P_{w g, i}^{k} \leq P_{w g . \max , i}^{k} \\
& P_{d g . \min , i}^{k} \leq P_{d g, i}^{k} \leq P_{d g . \max , i}^{k} \\
& Q_{d g . \min , i}^{k} \leq Q_{d g, i}^{k} \leq Q_{d g . \max , i}^{k} \\
& P_{n d . \min , i}^{k} \leq P_{n d, i}^{k} \leq P_{n d . \max , i}^{k} \\
& Q_{n d . \min , i}^{k} \leq Q_{n d, i}^{k} \leq Q_{n d . \max , i}^{k} \\
& P_{m . \min , i}^{k} \leq P_{m, i}^{k} \leq P_{\operatorname{m.max}, i}^{k} \\
& Q_{m . \min , i}^{k} \leq Q_{m, i}^{k} \leq Q_{m . \max , i}^{k} \\
& P_{c l . m i n, i}^{k} \leq P_{c l, i}^{k} \leq P_{c l . m a x, i}^{k} \\
& Q_{c l . m i n, i}^{k} \leq Q_{c l, i}^{k} \leq Q_{c l . m a x, i}^{k} \\
& P_{\text {ch.min }, i}^{k} \leq P_{\text {ch,i }}^{k} \leq P_{\text {ch.max }, i}^{k} \\
& E_{s, \min , i}^{k} \leq E_{s, i}^{k} \leq E_{s . \max , i}^{k} \\
& \lambda_{i j}^{k} \leq \lambda_{i j . \max }^{k} \\
& \omega i . \text { min }^{k} \leq \omega i^{k} \leq \omega i . \max ^{k} \\
& S_{i j}^{k} \leq S_{i j . \max }^{k} \\
& k \in[t: t+T-1]
\end{aligned}
$$

The cost function may include other objectives, such as conservation voltage reduction (CVR) and line losses however, in this study, since the distribution system is operated as a micro-grid, the objective function contains only the total generation and operation cost.

\section{Real-Time Implementation Challenges and Methodology}

Three main challenges related to real-time implementation of OPF are a) the need for fast convergence, $b$ ) computational speed with accurate results and c) ability to include sensor and estimated data from the field. In the proposed methodology, due the dynamic convex OPF implementation within RHC, the approach is computationally fast and activated at each time step there by feasible to include data at each time step. The results are accurate due to the proposed convexification approach. We report the simulation time based on Intel 2.4 GHz Core i5 CPU.The optimization platform is GAMS and SOCP solver is used to implement the formulation.

\section{CASE STUdies AND Simulation Results}

The 32-bus radial distribution system [25] is considered here to examine the convex RHC method. The system is $12.66 \mathrm{kV}$, and contains 33 buses (bus 0 is the contact point to upstream network) and 32 lines. The system is modified to incorporate the market transaction, wind generation, and storage (see Fig. 4). This radial network is connected to the upstream network and neighboring DISCO through tie lines from bus 0 and bus 30 respectively. Two wind farms are located at bus 13 and 16. One storage station is connected to bus 20. Contracted loads are considered at bus 7, 13, 24, and 31. Other system data are given in Appendix A and [25]. In this study, the
TABLE I

Total Cost Function [\$] At Differnet Time Step

\begin{tabular}{|c||c|c|c|c|}
\hline Step $(k)$ & 1 & 8 & 16 & 24 \\
\hline \hline RHC-SOCP & 81604 & 82643 & 84237 & 84721 \\
\hline RHC-ACOPF & 82440 & 83477 & 85075 & 85562 \\
\hline
\end{tabular}

wind generation is considered as a stochastic variable; i.e. the expectation of wind generation at time step $k$ of horizon window $[t, \ldots, k, \ldots, t+T-1]$ is changing by moving one step forward, $t$ to $t+1$. The actual and forecasted wind data from the electric reliability council of Texas (ERCOT) is used for the real time wind estimation at each time step. The time horizon $T$ is considered $24 h$, and each time step is considered $1 h$. However, since the optimization problem is solved in the order of seconds, it is viable to reduce the time step to less than a minute. Four case studies are considered to test the effectiveness of developed RHC-SOCP-OPF method.

A: Day ahead scheduling using ACOPF.

B: Day ahead scheduling using SOCP-OPF.

C: Real-time scheduling using RHC-ACOPF.

D: Real-time scheduling using RHC-SOCP-OPF.

Fig. 5 depicts the active power generation at bus 16 using convex methods A to D. It can be notified that the day ahead scheduling (method A and B) over estimated and under estimated wind generation close to $1 \mathrm{MW}$ at different time steps, compared to RHC methods (method C and D). It is noticeable that both methods $\mathrm{C}$ and $\mathrm{D}$ presented same optimal scheduling at bus 16 due to low wind price causing the wind generators are dispatched as higher priority. This also allows the wind to operate at it's maximum capability. This weakness of day ahead scheduling is shown in Fig. 6 in which the optimization methods A and B under estimated the power exchange with upstream network in hours 13 to 19. According to real-time data, wind is decreased during these hours. This reduction causes the increase in power exchange with upstream network represented in the RHC methods C and D. The Fig. 7 explains the charging and discharging schedule of storage station at each time step in convex RHC method. The storage is charging during hours 1 to 10 and then discharge from 13 to 21. This output is explained with the price pattern. In this system, the spot market price is higher during the discharging hours rather than charging period.

The performance of real-time methods $\mathrm{C}$ and $\mathrm{D}$ are compared in Fig. 8. The proposed convex method (D) results in significant reduction of the cost function in all steps of RHC implementation time steps. The reduction is close $\$ 1000$ and it is achieved at all steps 1 to 24 . This improvement is resulted from convex nature of proposed method $\mathrm{D}$, which guarantees the global optimum solution. Table I provide the total cost function value at time steps $1,8,16,24$, from which it can be seen that method $\mathrm{D}$ yields to $1 \%$ reduction in cost by finding the optimal schedule of the micro-grid. The other important aspect of the optimization methods is time performance. Table II illustrates the time performance of real time methods C and $\mathrm{D}$ at each time step. The proposed method D shows a significant reduction in simulation time, and yet converged to 


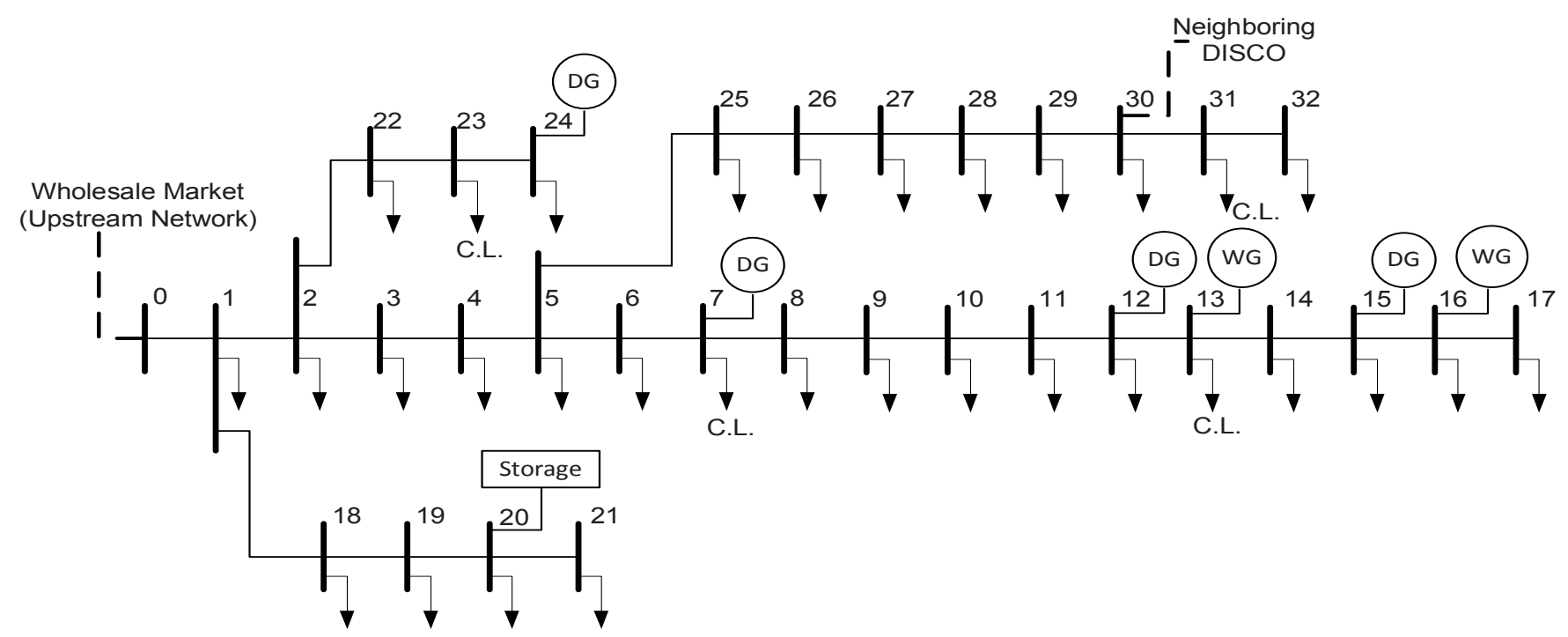

Fig. 4. IEEE 32 Bus Distribution Test System

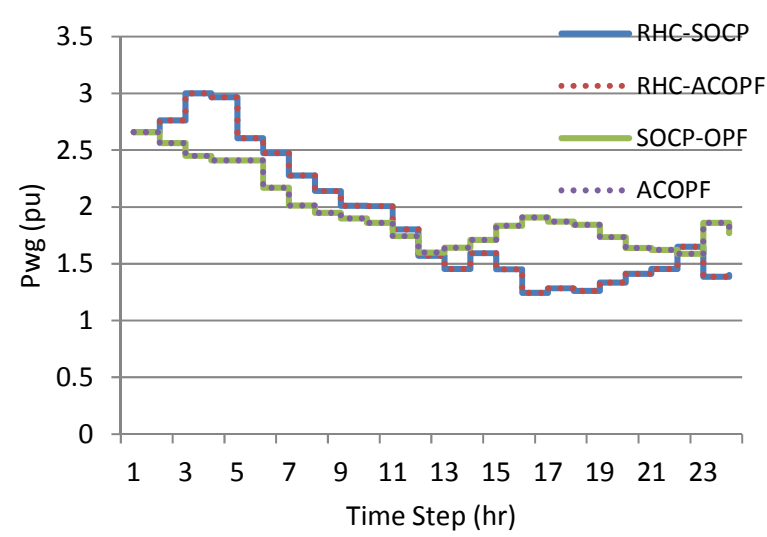

Fig. 5. Optimal Wind Schedule at Bus 16

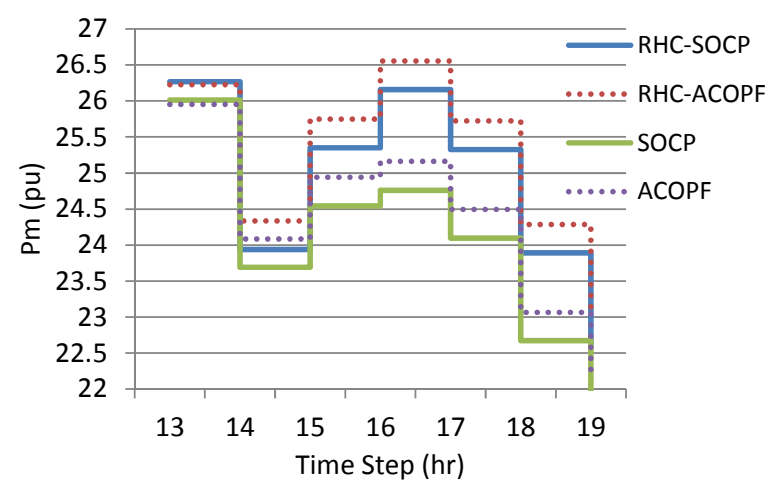

Fig. 6. Power Exchange with Upstream Network

TABLE II

SIMULATION TIME COMPARISON

\begin{tabular}{|c|c|}
\hline RHC-SOCP Average Simulation Time (Sec) & 6.8333 \\
\hline \hline RHC-ACOPF Average Simulation Time (Sec) & 14.2751 \\
\hline
\end{tabular}

the optimal solution in comparison with non-convex method $\mathrm{C}$ which yields to a local optimum solution. The average

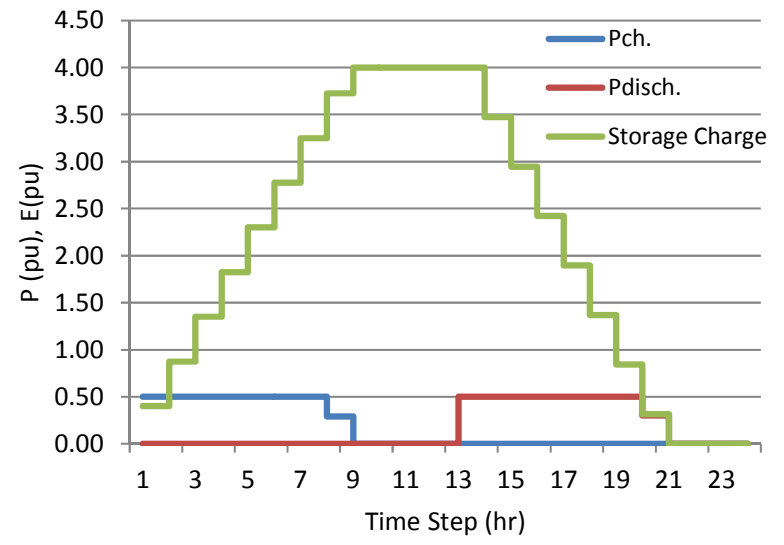

Fig. 7. Optimal Storage $P_{c h}$ and $P_{D i c h}$ From Convex RHC Method

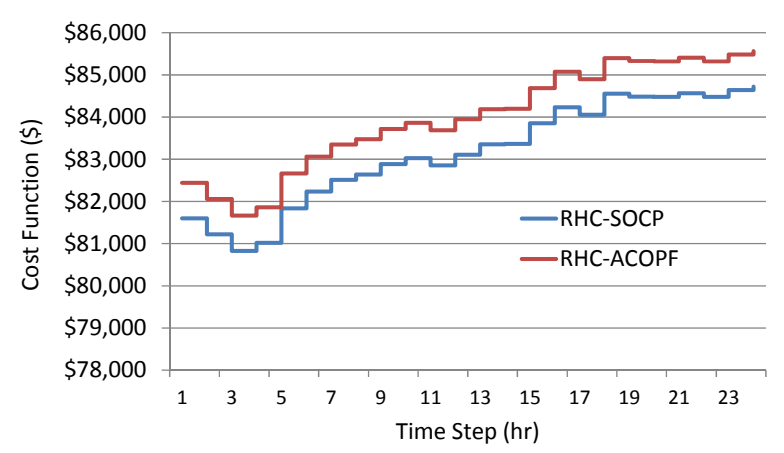

Fig. 8. Total Cost Over The Time Horizon

simulation time decreases from $14.275 \mathrm{sec}$ to $6.833 \mathrm{sec}$.

\section{CONCLUSiON}

We propose a real-time optimal power scheduling method for radial smart distribution system including dynamic components, market transactions, contracted loads and renewable generation. This convex RHC method takes into account the system uncertainties such as wind through re-estimating the 
TABLE III

Price Variation Coefficient At Differnet Time Steps

\begin{tabular}{|c||c|c||c||c|c|}
\hline Step $(k)$ & Price Coe. & Load & Step $(k)$ & Price Coe. & Load \\
\hline \hline 1 & 0.7 & 0.88 & 13 & 1.31 & 1.38 \\
\hline 2 & 0.64 & 0.83 & 14 & 1.46 & 1.41 \\
\hline 3 & 0.62 & 0.80 & 15 & 1.66 & 1.44 \\
\hline 4 & 0.59 & 0.78 & 16 & 1.43 & 1.45 \\
\hline 5 & 0.62 & 0.80 & 17 & 1.80 & 1.43 \\
\hline 6 & 0.63 & 0.85 & 18 & 1.43 & 1.39 \\
\hline 7 & 0.65 & 0.92 & 19 & 1.18 & 1.39 \\
\hline 8 & 0.65 & 1.02 & 20 & 1.14 & 1.34 \\
\hline 9 & 0.77 & 1.11 & 21 & 1.07 & 1.28 \\
\hline 10 & 0.84 & 1.20 & 22 & 0.92 & 1.15 \\
\hline 11 & 0.95 & 1.28 & 23 & 0.85 & 1.02 \\
\hline 12 & 1.09 & 1.34 & 24 & 0.80 & 0.94 \\
\hline
\end{tabular}

future inputs based on the real time data, which is repeated at each time step over the horizon window. The proposed method also address the major weakness of real-time methods based on non-convex ACOPF, referred as the local optimum solution and time inefficiency. The convex OPF is formulated within the RHC structure through two step relaxation and the second order conic programming (SOCP). The method effectiveness examined on the 32 bus test system, and the results shows the significant improvements in comparison with the current methods.

\section{ACKNOWLEDGEMENTS}

The authors gratefully acknowledge the support from National Science Foundation through NSF Grant ECS-1309911 and Grant ECS-1063484 awarded to the second author.

\section{APPENDIX A}

The market price at each time step is the multiplication of market price variation coefficient and the base price. The load demand variation at different nodes are calculated with the same procedure. DISCO optimize its operation schedule based on the energy market price in upstream, neighboring DISCO, wind variation, etc. Neighboring DISCO and upstream networks are capable to exchange power with the test system based on the hourly energy price. The base market price, and neighboring price are considered $\$ 0.09 / \mathrm{kWh}$ and $\$ 0.0825 / \mathrm{kWh}$ consequently. The variation coefficient of energy price with respect to base price are presented in Table III. The real-time wind forecasts over the horizon window are obtained at each time step from ERCOT wind forecast data [26].

\section{REFERENCES}

[1] P. J. Werbos, "Computational intelligence for the smart grid-history, challenges, and opportunities," Computational Intelligence Magazine, IEEE, vol. 6, no. 3, pp. 14-21, 2011.

[2] J. Carpentier, "Contribution to the economic dispatch problem," Bulletin de la Societe Francoise des Electriciens, vol. 3, no. 8, pp. 431-447, 1962.

[3] M. Huneault and F. Galiana, "A survey of the optimal power flow literature," Power Systems, IEEE Transactions on, vol. 6, no. 2, pp. 762-770, May 1991.
[4] J. A. Momoh, M. El-Hawary, and R. Adapa, "A review of selected optimal power flow literature to 1993. part i: Nonlinear and quadratic programming approaches," IEEE transactions on power systems, vol. 14, no. 1 , pp. 96-104, 1999.

[5] — "A review of selected optimal power flow literature to 1993. part ii: Newton, linear programming and interior point methods," IEEE Transactions on Power Systems, vol. 14, no. 1, pp. 105-111, 1999.

[6] K. Pandya and S. Joshi, "A survey of optimal power flow methods." Journal of Theoretical \& Applied Information Technology, vol. 4, no. 5, 2008.

[7] J. Lavaei and S. H. Low, "Zero duality gap in optimal power flow problem," Power Systems, IEEE Transactions on, vol. 27, no. 1, pp. 92-107, 2012.

[8] R. A. Jabr, "Radial distribution load flow using conic programming," Power Systems, IEEE Transactions on, vol. 21, no. 3, pp. 1458-1459, 2006.

[9] M. E. Baran and F. F. Wu, "Optimal capacitor placement on radial distribution systems," Power Delivery, IEEE Transactions on, vol. 4. no. 1 , pp. $725-734,1989$.

[10] - "Optimal sizing of capacitors placed on a radial distribution system," Power Delivery, IEEE Transactions on, vol. 4, no. 1, pp. 735743, 1989.

[11] J. A. Taylor, "Conic optimization of electric power systems," Ph.D. dissertation, Massachusetts Institute of Technology, 2011.

[12] J. A. Taylor and F. S. Hover, "Convex models of distribution system reconfiguration," Power Systems, IEEE Transactions on, vol. 27, no. 3, pp. 1407-1413, 2012.

[13] M. Farivar, C. R. Clarke, S. H. Low, and K. M. Chandy, "Inverter var control for distribution systems with renewables," in Smart Grid Communications (SmartGridComm), 2011 IEEE International Conference on. IEEE, 2011, pp. 457-462.

[14] M. Farivar, R. Neal, C. Clarke, and S. Low, "Optimal inverter var control in distribution systems with high pv penetration," in Power and Energy Society General Meeting, 2012 IEEE. IEEE, 2012, pp. 1-7.

[15] M. Farivar and H. Steven, "Low. branch flow model: relaxations and convexification (part i)," IEEE Trans. on Power Systems, 2013.

[16] E. Dall'Anese, H. Zhu, and G. B. Giannakis, "Distributed optimal power flow for smart microgrids," 2013.

[17] J. A. Momoh, "Smart grid design for efficient and flexible power networks operation and control," in Power Systems Conference and Exposition, 2009. PSCE'09. IEEE/PES. IEEE, 2009, pp. 1-8.

[18] J. Mattingley, Y. Wang, and S. Boyd, "Code generation for receding horizon control," in Computer-Aided Control System Design (CACSD), 2010 IEEE International Symposium on. IEEE, 2010, pp. 985-992.

[19] M. Khanabadi, S. Moghadasi, and S. Kamalasadan, "Real-time optimization of distribution system considering interaction between markets," in Industry Applications Society Annual Meeting, 2013 IEEE. IEEE, 2013, pp. 1-8.

[20] M. Kraning, E. Chu, J. Lavaei, and S. Boyd, "Message passing for dynamic network energy management," arXiv preprint arXiv:1204.1106, 2012.

[21] J. Robertson, G. P. Harrison, and A. R. Wallace, "A receding-horizon opf for active network management," in Electricity Distribution (CIRED 2013), 22nd International Conference and Exhibition on. IET, 2013, pp. $1-4$.

[22] P. Fortenbacher, "Power flow modeling and grid constraint handling in power grids with high res in-feed, controllable loads, and storage devices," Ph.D. dissertation, Masters thesis PSL 1113, Power Systems Laboratory, ETH Zurich, Switzerland, 2011.

[23] A. Subramanian, M. Garcia, A. Dominguez-Garcia, D. Callaway, K. Poolla, and P. Varaiya, "Real-time scheduling of deferrable electric loads," in American Control Conference (ACC), 2012. IEEE, 2012, pp. 3643-3650.

[24] P. Patrinos, S. Trimboli, and A. Bemporad, "Stochastic mpc for realtime market-based optimal power dispatch," in Decision and Control and European Control Conference (CDC-ECC), 2011 50th IEEE Conference on. IEEE, 2011, pp. 7111-7116.

[25] M. E. Baran and F. F. Wu, "Network reconfiguration in distribution systems for loss reduction and load balancing," Power Delivery, IEEE Transactions on, vol. 4, no. 2, pp. 1401-1407, 1989.

[26] , "Electric Reliability Council of Texas (ERCOT)," http://http://www. ercot.com/mktinfo/index.html/, -, [Online]. 